https://doi.org/10.5719/aub-g/68.1/4

\title{
MUTATIONS DE L'ESPACE PUBLIC \\ DANS LES ANCIENS TISSUS - CAS DU PALIS (KSAR) SIDI BOUTKHIL DE AIN SAFRA, ALGÉRIE
}

\author{
HADJI ABDELKADER ${ }^{1}$, FELOUSSIA LAHCENE $^{2}$, MEDJADJ TAREK ${ }^{3}$
}

\begin{abstract}
The ancient fabrics of ancient palaces represented by ksour in the desert Algerian have architectural and urban characteristics similar to those of many cities along the sub-Saharan Africa, that researchers call "Saharan urbanism or desert." These features are related to public spaces within the palaces which are considered as strong urban elements that ensure the links between the different units of the fabric and structure of the palace where the social cohesion and exchanges between residents happens.

The ksar is a striking example, who lived the same circumstances, but this architectural heritage remains marginalized and not integrated in urban projects in the city, or supported by the local community in development plans to restore and improve living conditions in this area palace. The impact of natural factors and intervention of some residents of the palace to connect their homes to different networks without considering the architectural value of this type of heritage and introduction of new building materials by some residents to repair or rebuild their homes without taking into consideration the architectural elements characterizing the palace are analysed.

This state of the palace has led us to question the role currently played by traditional public spaces of ksar which are considered life arteries inside the it. How to act on the future of these spaces in order to preserve the identity expressed through each of these components?

Keywords : Palace (ksar), architectural heritage, traditional public spaces, mutations, Ain Sefra.
\end{abstract}

\section{Introduction}

La croissance rapide des villes après l'indépendance et le passage de l'habitat en ksar à l'habitat urbain contemporain ont créé de nouvelles conditions avec des conséquences sur les modes de vie, la sociabilité et la pratique des espaces publics traditionnels. Où une urbanité nouvelle s'est installée, contribue d'avantage aux contrastes

1 MC-B, Laboratoire: Techniques Urbaines et Environnement, Institut: Gestion des Techniques Urbaines, Université Mohamed Boudiaf, M'sila, Algérie, kada28300@yahoo.fr

2 MC-A, Laboratoire: Techniques Urbaines et Environnement, Institut: Gestion des Techniques Urbaines, Université Mohamed Boudiaf, M'sila, Algérie, feloussia.lah9000@ gmail.com

3 MC-B, Institut: Gestion des Techniques Urbaines, Université Mohamed Boudiaf, M'sila, Algérie, medjadj_tarek@yahoo.fr 
entre l'habitat traditionnel autochtone et l'habitat moderne dynamique et ambitieux, dont la prospérité fait plaisir aux habitants. Cet état a donné une urgence particulière pour remédier le problème de la protection du patrimoine architectural des ksour menacés.

Cet intérêt porté à l'architecture traditionnelle provoque le respect de cet héritage culturel en matière d'identité architecturale.

L'Algérie a bénéficié d'un patrimoine architectural et urbain considérable, riche et diversifié, qui s'exprime tant dans l'urbanisme et les monuments des capitales impériales qu'à travers toutes les régions du pays. Ce type de patrimoine est jugé comme élément représentatif de l'historique architectural (Loi algérienne 98-04 relative à la protection du patrimoine). Les recherchent sur le patrimoine architectural sont élargies et diversifiées thématiquement aussi. Nous tentons à travers cet article de porter un regard sur l'état du Ksar d'Ain Safra et particulièrement sur les espaces publiques qui ont subi des mutations suite aux vagues de l'urbanisation dite moderne.

En générale, le patrimoine est une ressource symbolique étroitement liée à la question de la mémoire et de l'identité, mais également considéré comme une ressource économique de part de son intérêt qui porte une dimension touristique, comme le cas de plusieurs patrimoines architecturaux et urbanistiques tels que ksar de Boussemghoun situé au Sud-ouest d'Alger, et à $190 \mathrm{Km}$ au Sud-ouest d'El Bayadh et qui présente des avantages touristiques d'une qualité et d'un potentiel touristique très important (M.H. Ait Saadi et autres, 2017).

Le terme patrimoine signifie «l'héritage commun d'une collectivité, d'un groupe humain » (Petit Larousse, 1992).

«La notion de patrimoine couvre des espaces plus vastes relevant à la fois du monumental et du quotidien, de l'exceptionnel et de l'ordinaire » (Leniaud, 2002).

Les Ksour («Palais » en arabe, Ksar au singulier et Ksour au pluriel), « constituent un riche et diverse patrimoine architectural attractif et certaines, dont les quatre villes anciennes de Mauritanie, sont inscrites sur la liste du patrimoine mondial de l'humanité » (Maria Garvari-Barbas, 2013). Leur valeur patrimoniale est exprimée dans de nombreuses recherches liées à l'urbanisme. Cet héritage urbain demeure tout de même référencié aux monuments historiques, qu'il se doit de protéger et de transmettre « léguer un patrimoine préservé aux futures générations » (Le turc, 2001).

\section{Objectifs}

L'objectif principal de cette étude est d'éclaircir le coté spatial et social que représente l'identité de ce type de patrimoine.

L'intervention des collectivités locales mené sur le ksar (construction d'un mur de clôture) reste non encourageante et permettra aux propriétaires de rejoindre leurs demeures. 
Pour assurer cet objectif, on doit intervenir sur ce ksar de manière à revaloriser ce dernier à travers un type d'intervention adéquat et durable ; nous devons assurer un développement durable axé sur le secteur culturel, permettant de développer l'économie locale, le tourisme culturel, l'artisanat et valoriser les pratiques de la culture traditionnelle, en se référant à des expériences bien réussites (Casanovas et al, 2012).

\section{Approche d'analyse}

Selon (Arnold. M, 1984) «il serait mal venu d'attribuer à une théorie l'échec dans le traitement d'un problème si la théorie ne convient pas au type de problème considéré».

Après avoir fait un état des lieux sur la base des données du contexte urbain du corpus de recherche suite à un constat visuel basé sur l'observation en situation et les plans et schéma relatives à notre cas d'étude, nous allons utiliser l'approche morphologique qui est convenable à ce type de recherche afin de décrire le contexte historique des différentes phases de l'évolution du palais dit «Ksar ».

\section{Etudes antérieures}

Dans notre recherche, nous avons analysé des études antérieures qui se sont focalisées dans leurs ensembles sur les formes urbaines dans des villes anciennes (rues ruelles, places, placettes, squares et jardins publics) selon (Rémy Allain, 2004) " ces formes urbaines ont connus des transformations d'échelle et de nature qui les rendent plus complexes que jamais ». Ces études vont apporter une richesse à notre recherche et aider à la compréhension thématique de l'approche d'analyse, comme elles vont nous permettre d'ouvrir une fenêtre pour déterminer l'importance de l'étude menée qui constitue un facteur clé dans le développement des questions de la recherche et l'exploration du cas d'étude. Parmi ces études on souligne les suivantes :

\subsection{Première étude}

Il s'agit d'une étude intitulée Logique de conception et processus de production de l'espace public urbain (Philippe Hanocq, 2005), dont l'auteur démontre que l'évolution de ce qu'il nommera « espaces publics urbains ». Ce travail a mis l'accent sur les évolutions potentielles des espaces publics urbains, sous la pression conjuguée de facteurs qu'il perçoit comme sous-tendant fondamentalement le développement des sociétés urbaines dites postindustrielles. 
L'auteur a tenté de démontrer les concepts et les modèles concrets d'organisation des espaces publics urbains à travers quelques villes anciennes occidentales. Les facteurs qu'il a identifiés et retenus sont pris comme des principes paradigmatiques qui ouvrent un champ d'investigation vaste.

Dans la conclusion, l'auteur a parlé sur les pratiques sociales et spatiales des espaces publics dans la ville de Liège. Dans cette dernière, l'analyse morphologique a permet pour l'auteur de déboucher sur des résultats fondues et mieux étayées.

\subsection{Deuxième étude}

Il s'agit d'une étude basée sur la morphologie urbaine (Rémy Allain, 2014). Nous nous sommes limités aux points qui ont une relation étroite avec notre étude. Ces points se résument dans les formes urbaines et leurs significations dans le système urbain global.

L'étude se focalise sur une approche d'analyse et les différentes formes des espaces publics qui nous intéressent dans notre recherche. Selon l'auteur, cette réalité urbaine complexe analysable à différents niveaux d'échelle et de plusieurs points de vue, traverse les cloisonnements disciplinaires. Les études urbaines ont privilégié l'approche morphologique en mettant au centre de leurs préoccupations la question des formes urbaines.

L'auteur s'est posé la question, comment étudier et comprendre les formes urbaines sans se préoccuper des conditions historiques de leurs apparition ? il montre que la forme urbaine est la manifestation visuelle et partielle du système urbain et un de ses éléments les plus importants. Selon lui, l'étude morphologique est la clé de compréhension des formes urbaines, et que la forme urbaine est toujours perçue. C'est la position de Camillo Sitte et Raymond Urwin. L'auteur est arrivé à conclure que les différentes formes urbaines sont désormais au premier plan des préoccupations des aménageurs, des législateurs,

Ces études nous a permis de comprendre la thématique et l'approche d'analyse.

\section{Présentation du cas d'étude}

Dans notre étude intitulée Mutations de l'espace Public dans les anciens tissus. Cas du palais (ksar) d'Ain Sefra, on va étudier l'historique du ksar ainsi que les différentes mutations qui ont subi les espaces publics traditionnels à l'intérieur du ksar.

La ville d'Ain Sefra est entrée dans la division administrative conformément à la loi $34 / 09$. Elle située à $32^{\circ} 45^{\prime}$ latitude nord et à $0^{\circ} 35^{\prime}$ de longitude Ouest de Greenwich, à $440 \mathrm{~km}$ de la ville d'Oran, le centre de la ville est situé à 70 kilomètres au Sud du chef-lieu de la wilaya de Naama et occupe une 
superficie de $1045 \mathrm{~km}^{2}$ avec une population estimée à 43692 habitants en 2001 et une densité de population de 74ha / hab.

\subsection{Situation de la ville d'Ain Sefra}

La municipalité d'Ain Sefra est située dans le sud-ouest du chef-lieu de la wilaya et limitées (Figures 1), par les communes suivantes :

La commune de Naama et d'Ain ibn Khalil dans le nord ;

La commune de Tiout à l'est ;

La commune de Magrar dans le sud ;

La commune de Safesfa à l'ouest.

La ville d'A'in Sefra est le troisième pôle dans la wilaya de Naama après la commune de Naama et Mechria, comme elle a un réseau routier important constitué de la route nationale $\mathrm{n}^{\circ} 06$ qui assure le lien entre la wilaya de Béchar et Alger. La route nationale $n^{\circ} 47$ assure la liaison entre la ville d'Ain Sefra et la wilaya d'El-Bayadh.

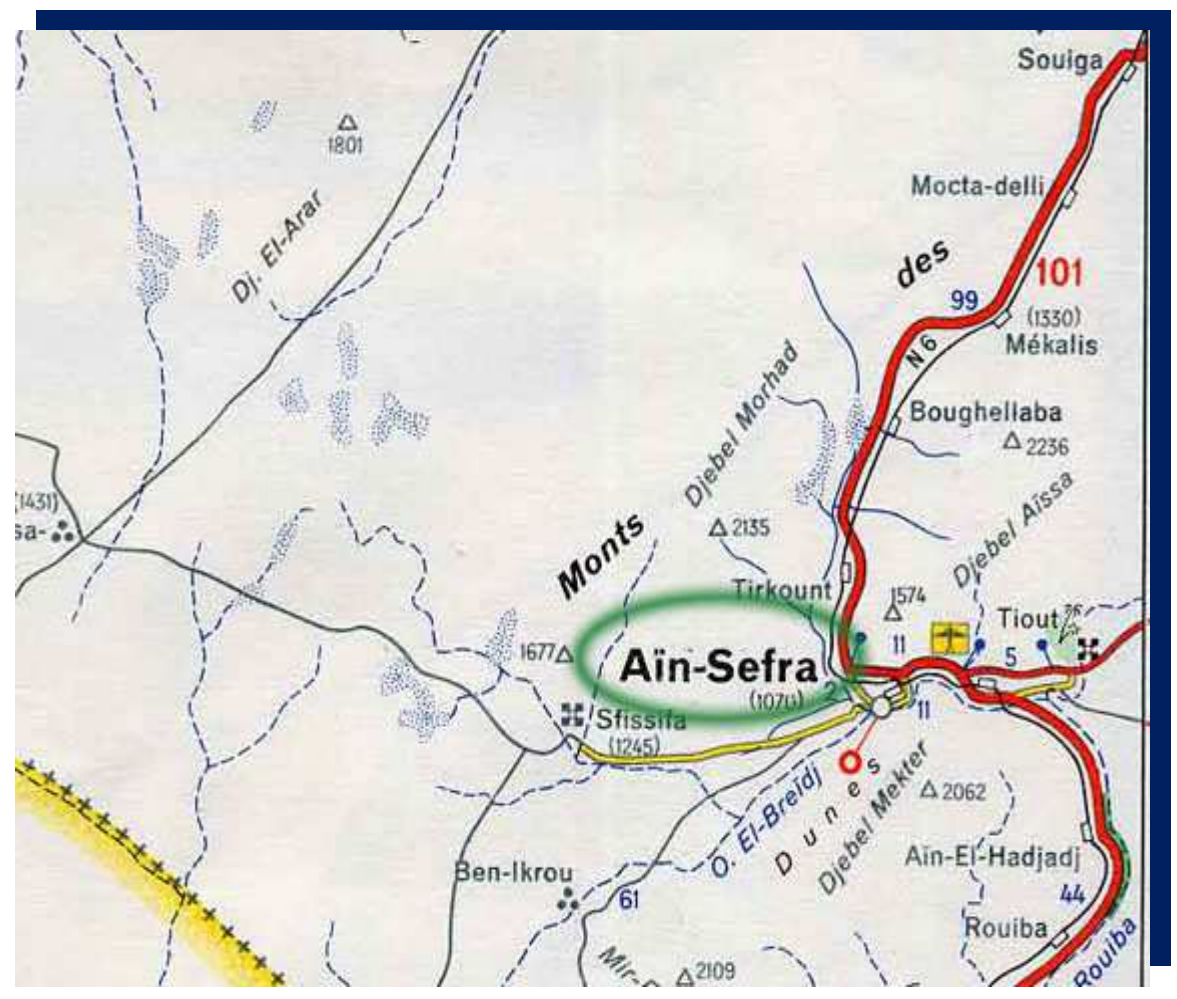

Fig.1. Localisation de la ville d'Ain- Sefra Source : Plan cadastral de la commune d'Ain-Sefra 
Plan de la ville d'Ain Sefra Ech: 1/50000
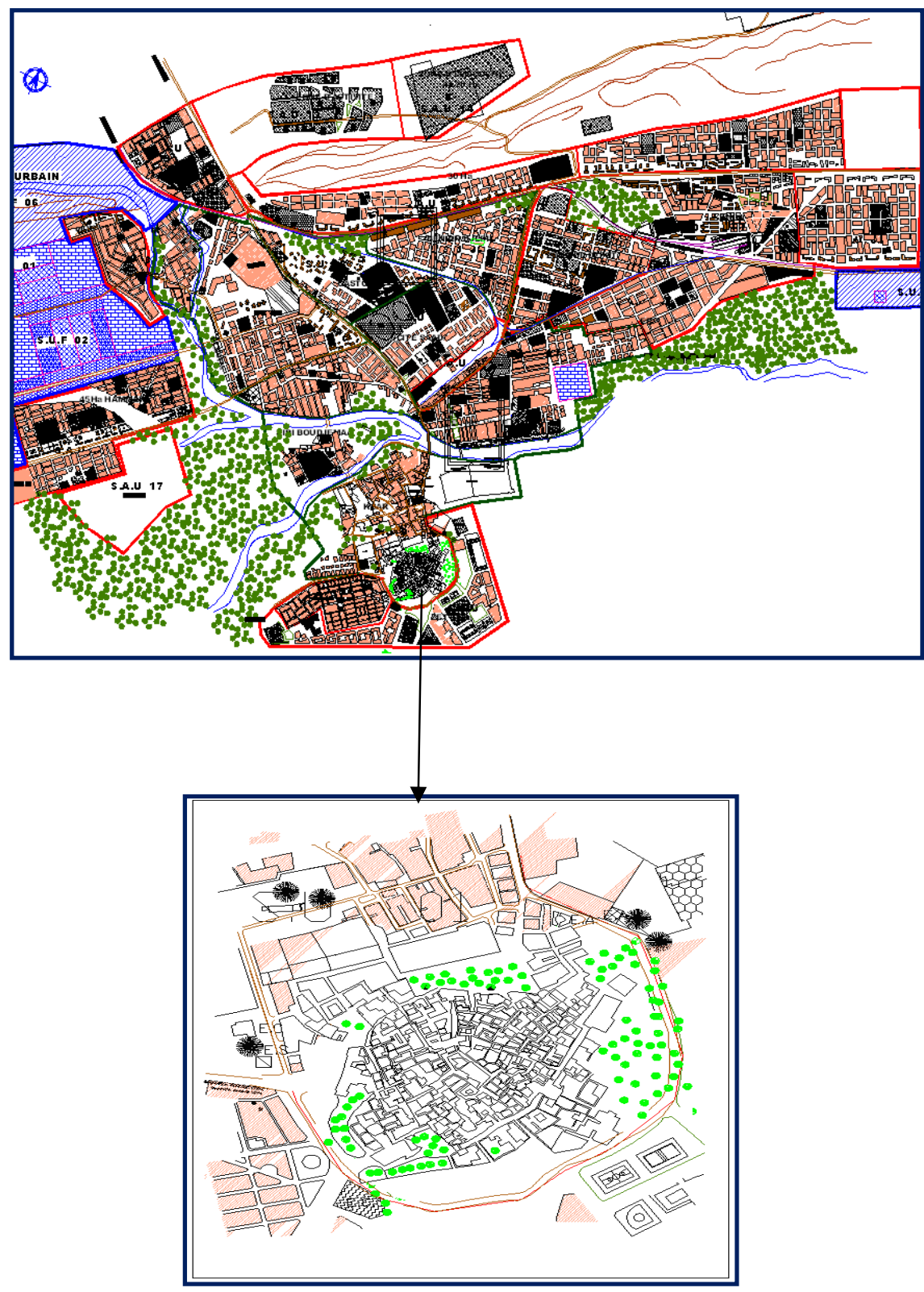

Le ksar

Source : Plan Directeur d'Aménagement et d'Urbanisme de la commune d'Ain Safra, 2012 
Le ksar est situé au Sud-est de la ville d'Ain Sefra et limité (Figures 2), au Sud par des dunes de sables et quelques constructions individuelles éparpillées, à l'Est par des équipements sportifs, à l'Ouest par une oasis de palmiers et un Oued et au Nord par une nouvelle extension de la ville.

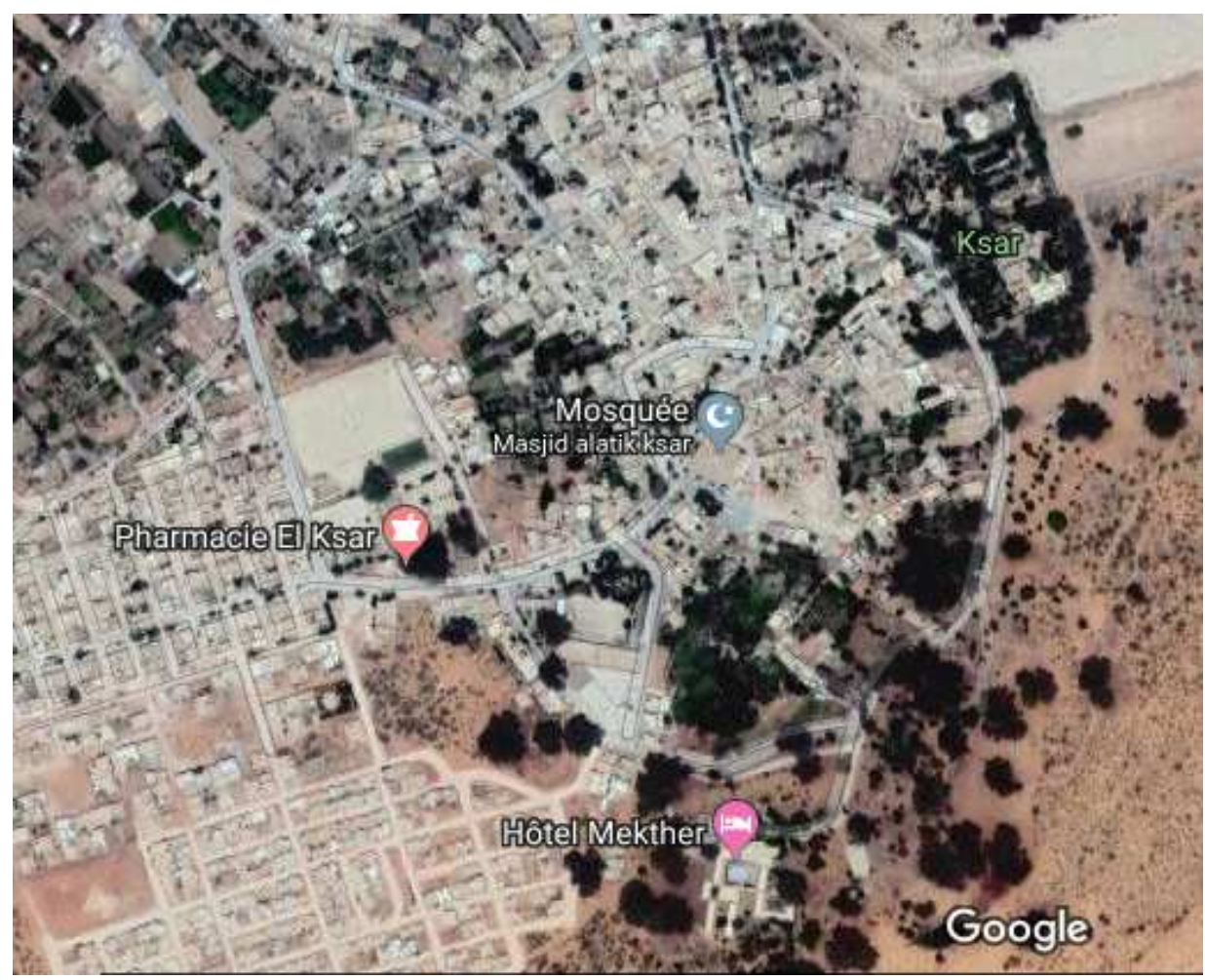

Fig. 2. Image satellitaire : limite de l'état actuel du ksar Source : Données cartographique, digital global 2019 Google

\subsection{Caractéristiques hydro-climatique de la ville d'Ain Sefra}

Les eaux souterraines constituent actuellement les principales ressources pour la ville d'Aïn Sefra qui représente la principale activité socio-économique de la région.

La ville d'Ain Sefra est dominée par un climat continental qui constitue l'avantage des villes de haute steppe en raison de la présence des montagnes qui empêchent l'entrée d'influences marines de l'océan Atlantique

Les influences venant de la mer Méditerranée sont peu nombreuses, en raison de l'étroitesse du front de mer même les influences du désert ne pénètrent 
pas dans la région en raison de la présence de l'atlas du désert qui est caractérisée par un été chaud et sec et un hiver froid; ce climat désertique se présente à travers le diagramme ci-dessous.

\subsubsection{Diagramme climatique d’Aïn Séfra}

Les précipitations varient entre le mois le plus sec et le mois le plus humide de $18 \mathrm{~mm}$ à $22 \cdot 1^{\circ} \mathrm{C}$ qui s'affichent sur l'ensemble de l'année (Figure 3 ).

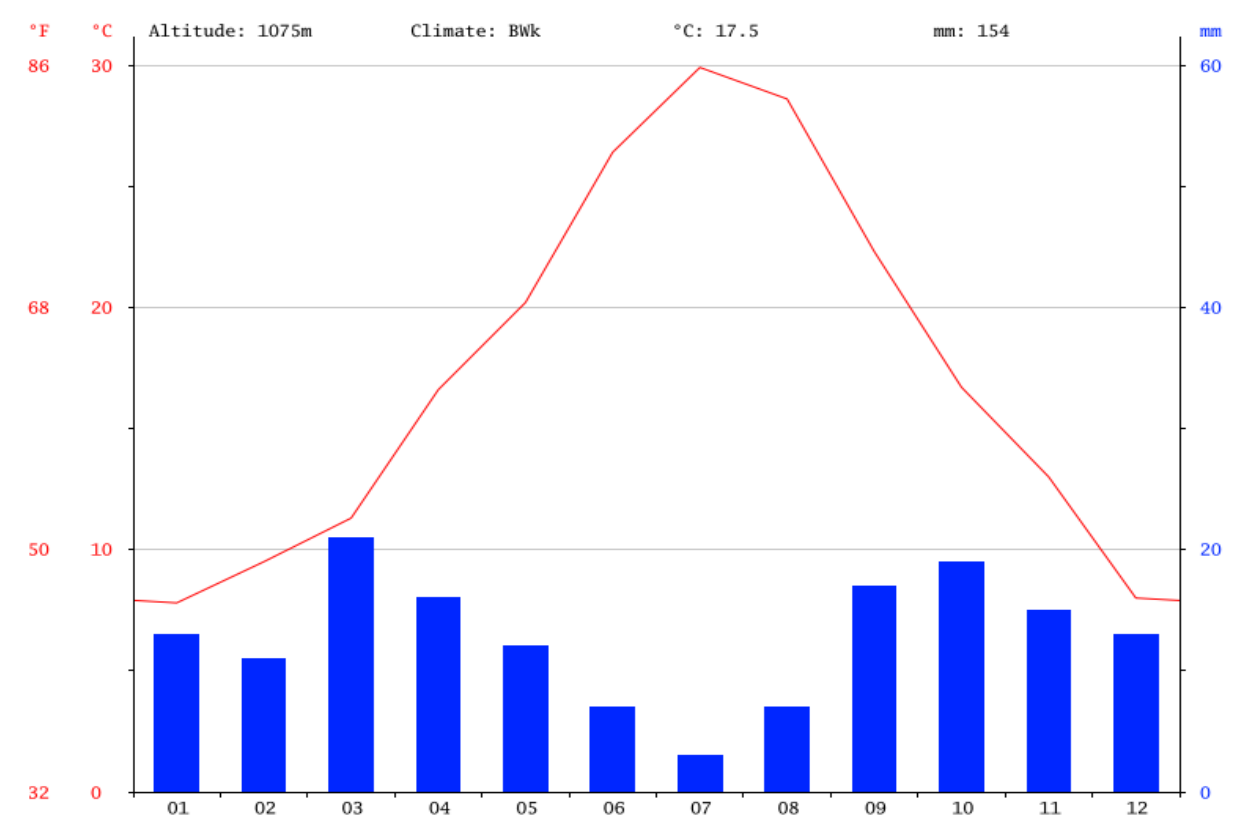

Fig. 3. Diagramme climatique d'Aïn Séfra

Source : https://fr.climate-data.org/afrique/algerie/naama/ain-sefra

\subsection{Signification du mot Ksar}

Dans la littérature arabe concernant ce type de patrimoine architectural traditionnel, on désigne par ce mot à une altération de la racine arabe, un espace d'habité qui est court et limité, auquel n'a accès que pour certaine catégorie de groupe sociaux ; c'est un espace confiné et réservé à l'usage de certains. Le ksar selon Moussaoui. A (1994) dans son doctorat, est un grenier, mieux encore un ensemble de greniers bien ajoutés. 


\subsection{Identification du Ksar en tant que bâti}

Le Ksar est un reflet d'histoire où s'exprime une identité culturelle et une intégration très forte dans l'environnement ; on constate par ailleurs, en référence au concept d'identité une très forte cohérence du domaine bâti; reflet logique et évident du concept d'appartenance au ksar, ce dernier est caractérisé par une grande capacité d'isolation thermique qui réside selon les habitants dans le matière de construction qui est la brique à base d'argile et la conception organique des cellules de maisons.

\subsection{Caractéristiques du Ksar}

Parmi les caractéristiques du Ksar, nous noterons les éléments suivants :

- Sa préservation du style architectural employant la terre compactée comme matériaux de construction ;

- Son adaptation avec les conditions climatiques et environnementales ;

- Son harmonie avec le milieu environnemental ;

- La cohérence de ses parties et ses dimensions architecturales ;

- L'authenticité des méthodes architecturales utilisées, témoignant des modes de vie en vigueur dans une région désertique.

\subsection{Morphologie du Ksar (Etat des lieux)}

Le Ksar est une forme d'habitat qui constitue un tissu organique et compact qui prend la couleur de la terre du site et qui est en relation avec la palmeraie. Selon l'échelle d'appropriation, le ksar se compose de trois entités urbaines qui sont :

Les demeures s'allongent le long d'une ruelle sinueuse appelée «Zkak », ou autour d'une placette qui prend le nom de «Rahba».

Des espaces publics traditionnels collectifs (ruelles, placettes et espace semi-collectif) qu'est l'impasse « Derb » qui signifie voie sans issue.

L'ensemble de ces formes urbaines traditionnels sont articulés selon une hiérarchie spatiale et fonctionnelle; ce principe est similaire pour l'ensemble des Ksour (addition de plusieurs ksar) subsahariens. Le Ksar est entouré d'un rempart qui a pour but la défense contre les ennemis. Ce produit d'articulation est caractérisé par des cellules de maisons qui ont presque la même configuration spatiale planimétrique et altimétrique avec une hauteur qui varie entre 02 à $02,5 \mathrm{~m}$ et une superficie moyenne de la chambre de 08 à $09 \mathrm{~m}^{2}$. 
Comme tous les ksour du sud Algérien, le ksar de sidi Botkhil présente un tissu organique, organisé en forme d'unités adjacentes des mêmes entités familiales, les maisons ont une couleur grise, bâties généralement en pierre, possédant une cour intérieure et un étage: Ces maisons présentent des caractéristiques typologiques uniformes dont les formes urbaines traditionnelles sont disposées selon un modèle d'organisation hiérarchisé qui convergent vers le centre suivant une maille de ruelles tortueuses et obscures; le tout constitue un schéma de structure radioconcentrique.

\subsection{Evolution spatiale du Ksar d'Ain Sefra}

Le Ksar d'Ain-Sefra fût créé vers 1586 soit vers l'an 987 de l'Hégire par Mohamed Ben-Chaïb - dit Bou-DekhiL et ses enfants qui ne sont pas d'origine berbère mais sont issus d'éléments divers de race arabe. Au début, le ksar était divisé en deux parties et bâti entre les dunes et l'oued non loin de la source d'eau, abritant la population arabe locale. Il est adossé à une grande ligne de dunes d'environ15 kilomètres de long qui le sépare du Djebel Mekter.

Selon le Sénat de la ville d'Ain-Sefra, l'organisation politique, administrative et judiciaire du Ksar, avant l'arrivée des Français, était administrée par un groupe de résidents âgés. En 1849, il comptait 260 maisons habitées par 120 familles. Parmi eux, 60 familles ont quitté le Ksar avant l'occupation française. Ce ksar a un tissu dense et organique (voir figure 4), l'ensemble des espaces urbains constituant le ksar s'organisent autour d'une mosquée. Historiquement, le poste d'Ain-Sefra fût créé en 1882, après l'insurrection de Bou-Amama, pour surveiller la région en face du ksar qui était, alors, la citadelle et le refuge des dissidents de la région. Durant la colonisation, l'agglomération d'Ain-Sefra fût rendue célèbre par le maréchal français Lyautey qui commanda la Subdivision de cette agglomération de 1903 à 1906.

Le barrage composé de pierres sèches retenues par des piquets solidement fixés à terre servant à l'irrigation des jardins, a été démoli. Actuellement, la plupart des résidents ont quitté le ksar à cause de son état déplorable ainsi que le manque d'intérêt des autorités locales pour ce type de patrimoine architectural. 


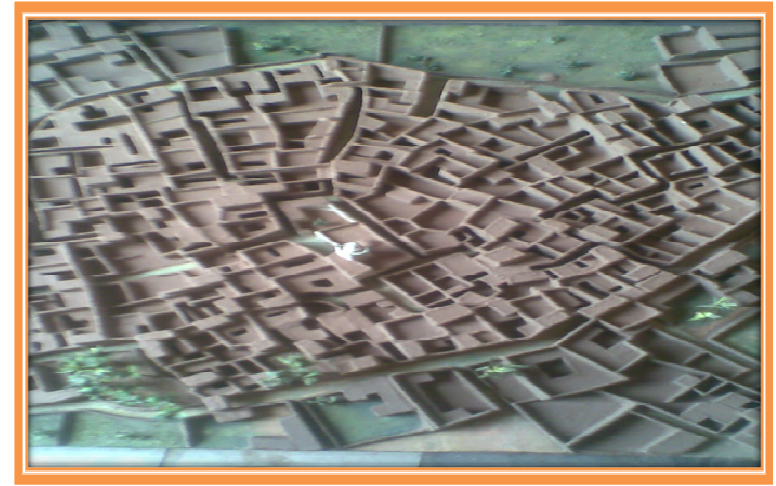

Fig. 4. La morphologie du ksar Ain Sefra selon une maquette Source : centre culturel d'Ain Sefra, 2010

Le tissu urbain traditionnel et organique est un résultat de création collective et spontané d'une société cohésive. Il se compose de :

\subsection{Le cadre bâti}

La morphologie du cadre bâti de ce Ksar se caractérise par une homogénéité dans la forme, dans les matériaux de construction, les conditions humaines et historiques, les pratiques sociales et l'appropriation de l'espace, qui ne font qu'enrichir l'unité traduite dans les relations humaines et autres règles qui structurent et régissent la société.

La préservation du style architectural utilise la terre compactée comme matériaux de construction proprement dit (figure 5).

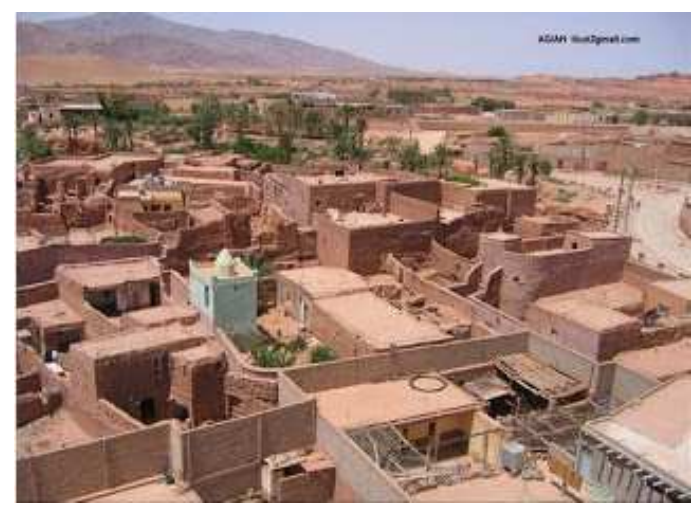

Fig. 5. Etat actuel du ksar

Source : Photo prise par les auteurs 


\subsection{Le cadre non bâti (espaces publics traditionnels)}

Le cadre non bâti est caractérisé par une trame vernaculaire avec un maillage de voies sinueuses et étroites qui donnent sur des espaces libres considérés comme artères de vie à l'intérieur de ce ksar, et qui sont :

\subsubsection{La rue}

C'est l'espace public par excellence. La rue se trouve à la périphérie du ksar, revêtue en macadam de pierre avec une inclinaison vers le milieu de la chaussée qui n'a pas de trottoir tout en créant un fil d'eau pour les eaux pluviales (figure 6). La rue est aussi le théâtre d'une multitude de pratiques sociales et spatiales à la fois. Les propriétaires des échoppes exposent leurs marchandises parfois sous forme d'un étalage en dehors de leurs devantures afin d'attirer le maximum possible d'acheteurs ; c'est là où se côtoient aussi les usagers.

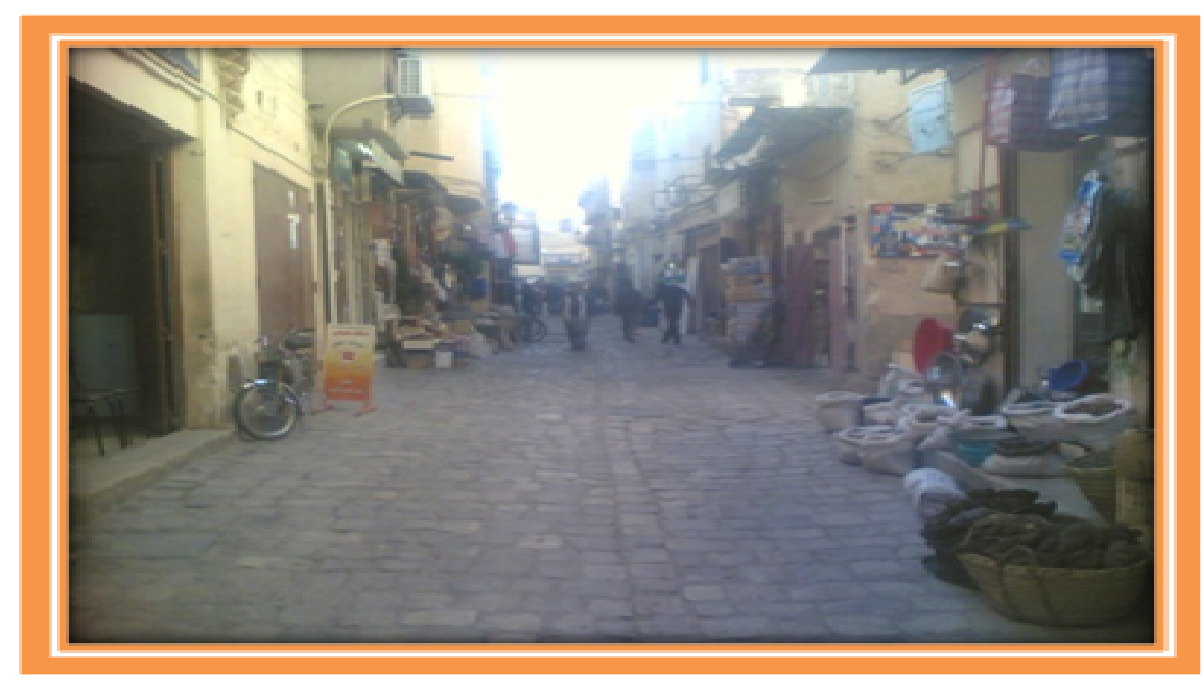

Fig. 6. Rue commerçante

Source : photo prise par les auteurs

\subsubsection{La ruelle}

Zkak ou Zenka est un espace public constitue d'un groupement d'habitation fermé (figure 7), avec une largeur qui est juste nécessaire au croisement de deux ânes bien chargés ; cet espace est bien codifié en particulier par les usagers masculins. 


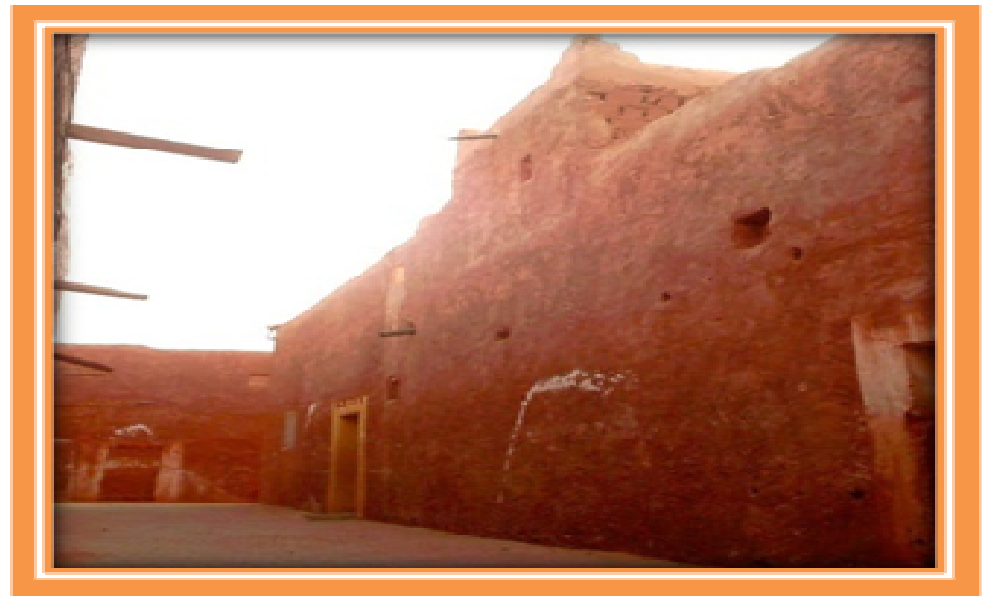

Fig. 7. La ruelle : effet de découvert Source : photo prise par les auteurs

La ruelle est une catégorie d'espace public très répondue qui a pour but de créer des dispositions spatiales afin de préserver l'intimité privé ; la ruelle n'était pas le résultat d'une croissance incontrôlée et anarchique mais consciemment planifiée.

Le processus d'appropriation et d'occupation de ce type d'espace dit semi-public peut être expliqué par l'installation successive de plusieurs occupants à travers le temps et qui ont tenu à préserver l'accès à leurs habitations.

\subsubsection{L'impasse}

L'impasse est un élément urbain essentiel qui fait partie de la trame viaire des médinas ou des villes traditionnelles; «il est l'élément de base de l'urbanisme musulman traditionnel, son apparition étant liée à un type d'habitat dans lequel la ville s' organise exclusivement autour d'une cour intérieure et qui ignore par conséquence la façade » (Raymond, A 1985, p. 193). Cette impasse s'appelle en langue arabe «Derb » et occupe une partie importante de la trame viaire du tissu du ksar avec une surface qui est estimée, suivant les études analytiques qui ont été faites sur quelques villes traditionnelles arabes par l'auteur précité, à Damas de 43,1\%, à Alger de 45,7\% et au Caire de 46,8\%.

Dans notre cas, cet espace se rapproche de $41 \%$; il n'y a accès que pour les propriétaires des maisons qui donnent sur cette impasse ou leurs proches (figure 8); cette caractéristique d'intimité est renforcée davantage par des entrées de maison qui ne sont jamais face à face afin d'éviter les entrées contiguës ou de vis-à-vis. 


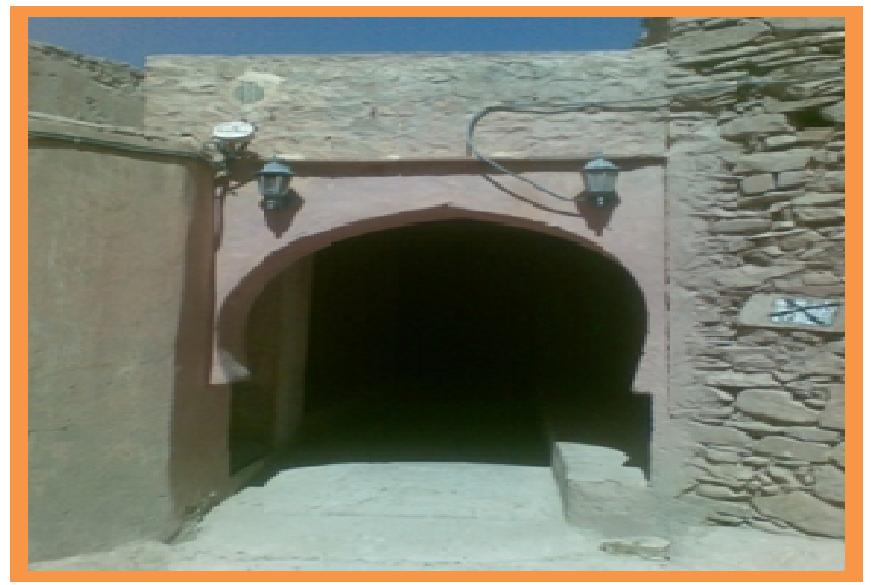

Fig. 8. L'impasse : « Derb»

Source : photo prise par les auteurs à l'intérieur du Ksar

Cet état de fait peut clarifier la logique d'utilisation et d'appropriation de ce type d'espace par les usagers. La configuration spatiale de cet espace peut prendre la forme d'entonnoir, ce dernier pouvant prendre aussi deux formes : soit un espace très réduit qui donne accès à une ou deux habitations ou à un espace en forme d'une petite cour sans issue dans laquelle s'ouvrent plusieurs accès de maisons. L'usage de cet espace ne peut en aucun cas être détourné à un autre usage sans l'avis préalable de l'ensemble des usagers.

\section{Conclusion}

Le Ksar (palais) d'Ain Sefra porte les traces de l'héritage des espaces publics maghrébins à forte connotation locale. Cette entité urbaine a subi une profonde mutation, en particulier celle liée aux artères du Ksar représentées dans les différentes formes de l'espace public urbain.

Les espaces publics se sont des lieux où se déroulent les différentes pratiques des habitants du ksar, telles que le commerce, la circulation, la détente, la rencontre et le côtoiement. Ces espaces subissent alors différentes mutations qui se lisent, tant dans leur valeur symbolique, qu'à travers leurs fonctions et leurs usages.

Il est évident qu'à partir de ce qu'on a développé sur ce type d'établissement humain qu'est le ksar, qu'une telle interprétation socio-historique de l'espace public relève d'un ordre social qui réglemente les différents rapports socioculturels qui existent à l'intérieur du ksar. En l'absence d'une politique nationale efficace en matière de préservation et de gestion du patrimoine architectural du ksar, l'état 
actuel de ce dernier à tendance de se dégrader. La sauvegarde de ce type de patrimoine est une opération qui nécessite non seulement de gros moyens humains et matériel mais aussi une volonté politique fondée sur une vraie politique de sauvegarde du patrimoine.

A ce propos, il est utile de mettre en valeur ces espaces publics traditionnels à travers :

- La mise à jour et l'activation des lois relatives à ce type de patrimoine ;

- Se disposer des moyens financiers afin de remédier les problèmes de tous les types de dégradations ;

- La requalification de ces espaces à travers une main d'œuvre qualifiée dans la restauration de ce type de patrimoine ;

- Affectation des diverses études et recherches à des consultants compétents ou d'ordre pluridisciplinaire afin d'affiner une piste de réflexion prometteuse sur les espaces publics et répondre aux enjeux à venir ;

- La mise en place d'un organisme national pour l'entretien, la surveillance et la gestion de ce patrimoine, en particulier les différentes formes de l'espace public urbain dans ce type de patrimoine ;

- Le Ksar doit bénéficier d'un programme d'aide qui permettra sa restauration partielle dans le respect des procédés anciens de construction ;

- La sensibilisation au patrimoine architectural représente un enjeu essentiel, et oriente en tant que stratégie à suivre dans différents niveaux d'intervention;

- Ce patrimoine doit rester étroitement lié à l'intérêt des populations locales et à l'implication active de la société civile et des communautés locales ;

- Donner de l'importance à ce patrimoine dans l'enseignement scolaire, universitaire et professionnel, également à la sensibilisation des administrateurs ;

- Promouvoir ce patrimoine à travers d'émissions radiophoniques et télévisées.

\section{BIBLIOGRAPHIE}

Ait Saadi, M.H., Remini, B., Farhi, A., 2017, Le ksar de Boussemghoun : Identification de son patrimoine et perspective de promotion du tourisme saharien, Courier du savoir, 23, pp. 55-74.

Arnold, M.R., 1984, Théorie et méthode en science sociale, Université de Minnesota presse, Paris.

Casanovas, et al., 2012, Montada: Patrimoine et participation; vers un nouveau cadre de gouvernance au Maghreb.

Garvari-Barbas, M., 2013, Habiter le patrimoine : Enjeux, approches, vécu, Rennes, publication sur Open Edition Books : 22 février.

Hanocq, Ph., 2005, Logique de conception et processus de production de l'espace public urbain, cas de la ville de liège en Belgique, thèse de doctorat en sciences appliquées, Université de Liège, Faculté des Sciences Appliquées.

Le Turc, A.S., 2001, De l'écologie urbaine au développement durable, dossiers d'experts, Haute-Normandie.

Leniaud, J.M., 2002, Les archipels du passé : le patrimoine et son histoire, Fayard, Paris. 
Moussaoui, A., 2006, Dans l'habitat oasien : espace et société, dans les actes du séminaire «Vivre dans les déserts : un aménagement urbain durable est-il encore possible dans les régions arides et chaudes ?» Ghardaïa, Algérie, 9-12 décembre.

Rémy, A., 2004, Morphologie urbaine, géographie, aménagement et architecture de la ville, édition. Armand Colin, Paris.

*** Décret exécutif n ${ }^{\circ}$ 91/175 du 28/05/1991, règles générales d'aménagement et d'urbanisme.

*** Données cartographique, digital global 2019 Google.

*** La loi 34-09,"La ville d'Ain-Sefra est entrée dans la division administrative conformément à la loi 34/09", Journal officiel de la république algérienne démocratique et populaire.

*** La loi 98-04, relative à la protection du patrimoine, Journal officiel de la république algérienne démocratique et populaire.

*** Plan directeur d'aménagement et d'urbanisme de la commune d'Ain Sefra, 2012.

*** Plan cadastral de la commune d'Ain-Sefra.

Petit Larousse illustré, 1992, Amazon, Paris.

https://fr.climate-data.org/afrique/algerie/naama/ain-sefra. 